\title{
Exoplanet properties from Lick, Keck and AAT
}

\author{
G W Marcy ${ }^{1}$, R P Butler ${ }^{2}$, S S Vogt ${ }^{3}$, D A Fischer ${ }^{4}$, J T Wright ${ }^{1}$, \\ J A Johnson ${ }^{5}$, C G Tinney ${ }^{6}$, H R A Jones ${ }^{7}$, B D Carter ${ }^{8}$, J Bailey ${ }^{9}$, \\ S J O'Toole ${ }^{10}$ and S Upadhyay ${ }^{1}$
}

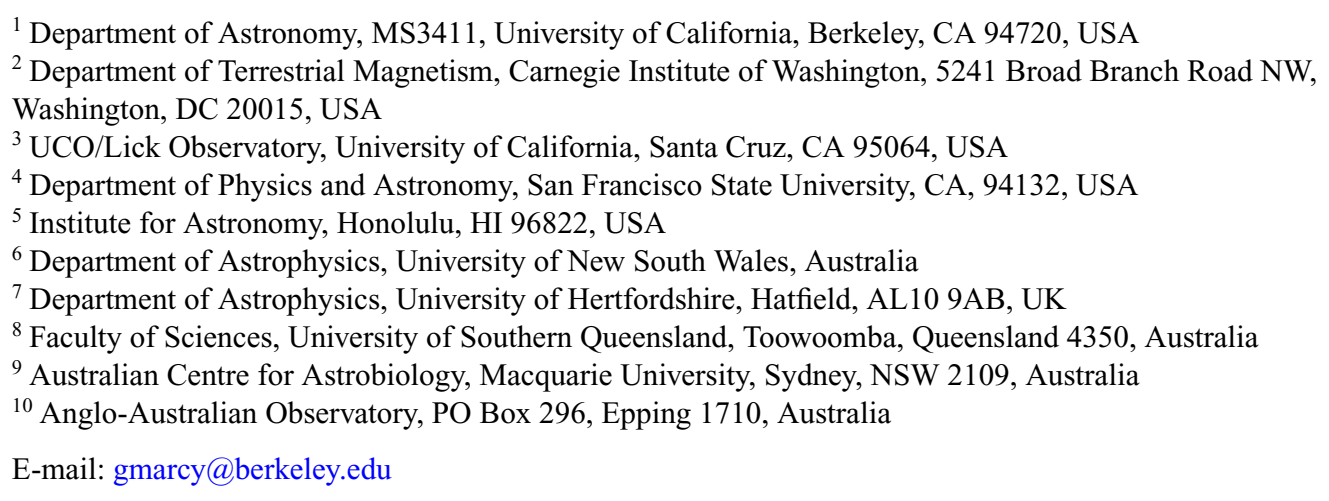

Received 11 March 2008

Accepted for publication 14 March 2008

Published 16 July 2008

Online at stacks.iop.org/PhysScr/T130/014001

\begin{abstract}
Doppler-shift measurements with a remarkable precision of $\Delta \lambda / \lambda=3 \times 10^{-9}$, corresponding to velocities of $1 \mathrm{~m} \mathrm{~s}^{-1}$, have been made repeatedly of 2500 stars located within 300 light years. The observed gravitational perturbations of the stars have revealed 250 orbiting planets, with 27 that cross in front of the host star, blocking a fraction of the starlight to allow measurement of the planet's mass, radius and density. Two new discoveries are the first good analog of Jupiter (HD 154345b) and the first system of five planets (55 Cancri). The predominantly eccentric orbits of exoplanets probably result from planet-planet gravitational interactions or angular momentum exchange by mean-motion resonances. The planet mass distribution ranges from $\sim 15 M_{\mathrm{JUP}}$ to as low as $\sim 5 M_{\text {Earth }}$ and rises toward lower masses as $\mathrm{d} N / \mathrm{d} M \propto M^{-1.1}$. The distribution with orbital distance, $a$, rises (in logarithmic intervals) as $\mathrm{d} N / \mathrm{d} \log a \propto a^{+0.4}$. Extrapolation and integration suggests that $19 \%$ of all Sun-like stars harbor a gas-giant planet within $20 \mathrm{AU}$, but there remains considerable incompleteness for large orbits. Beyond $20 \mathrm{AU}$, the occurrence of gas-giant planets may be less than a few per cent as protoplanetary disk material there has lower densities and is vulnerable to destruction. Jupiter-mass planets occur more commonly around more massive stars than low mass stars. The transit of the Neptune-mass planet, Gliese $436 \mathrm{~b}$, yields a density of $1.55 \mathrm{~g} \mathrm{~cm}^{-3}$ suggesting that its interior has an iron-silicate core surrounded by an envelope of water-ice and an outer $\mathrm{H}-\mathrm{He}$ shell. Planets with masses as low as five Earth-masses may be commonly composed of iron-nickel, rock and water along with significant amounts of $\mathrm{H}$ and $\mathrm{He}$, making the term 'super-Earth' misleading. The transiting planet HD147506b has high orbital eccentricity but no significant orbital inclination to the line of sight, presenting a puzzle about its history. Its orbit together with the mean motion resonances of 4 of the 22 multi-planet systems provides further evidence for the role of planet-planet interactions in shaping planetary architectures.
\end{abstract}

PACS numbers: 97.82.-j, 97.82.Fs, 97.82.Jw

(Some figures in this article are in colour only in the electronic version.) 


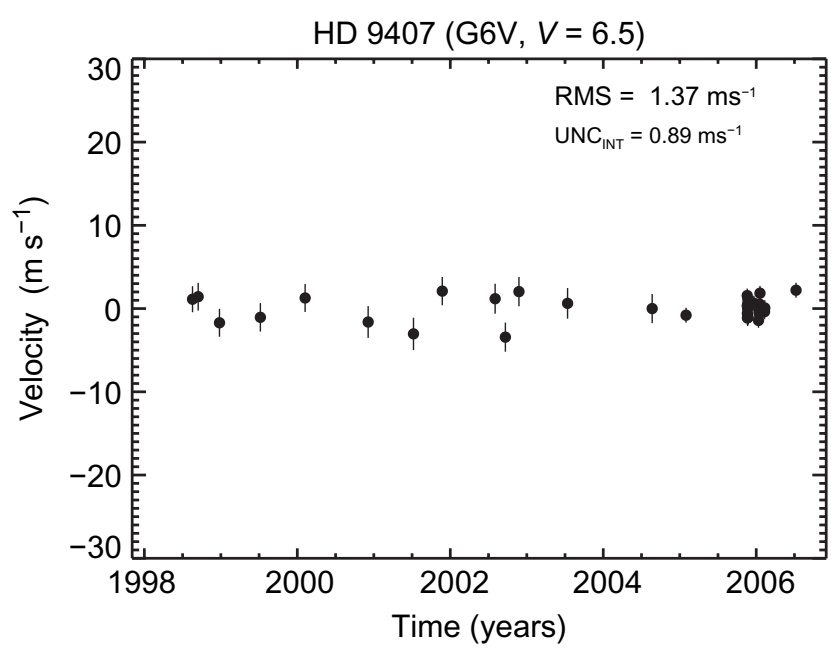

Figure 1. Velocity versus time for HD 9407 (G6 V) during 9 years observed at the Keck telescope with exposures of 1 min. The RMS of $1.37 \mathrm{~m} \mathrm{~s}^{-1}$ demonstrates the total error budget, including Poisson errors from the photons, any instrumental errors, photospheric velocity fields and any unknown small planets. The iodine technique thus has long-term errors under $1.5 \mathrm{~m} \mathrm{~s}^{-1}$ during 10 years.

\section{Doppler observations and properties of exoplanets}

Prior to 1995, no planets had been found around normal, hydrogen-burning stars, leaving our Solar System as the only known such planetary system, possibly a rare phenomenon in the universe. During the past 12 years, 250 planets have been discovered orbiting stars within 700 light years, the majority found by precise Doppler shift measurements of the host stars. The Doppler-detected reflex motion of the host star reveals the planet's orbital period, orbital distance, orbit shape and a lower limit on the planet mass, $M \sin i$ (where $i$ is the orbital inclination to the line of sight). These first exoplanets constitute a statistically useful ensemble of orbits and masses that exhibit distributions unexpected by previous theories of planet formation and the dynamics of their interactions.

Doppler shifts are now measured with precision of $\sim 1 \mathrm{~m} \mathrm{~s}^{-1}$ by teams in Geneva, Texas, Harvard, Japan, Tautenberg and also by our 'California and Carnegie Planet Search' (CCPS) and Anglo-Australian Planet Search (AAPS) groups that utilize the Lick, Keck and Anglo-Australian telescopes. Such Doppler precision amounts to a displacement of $7 \mathrm{~nm}$ on the CCD detector (a few dozen silicon atoms on the substrate), and the Doppler metric must be maintained at that nanometer level for durations of decades to span the orbital periods of planets. Extraordinary optical and algorithmic techniques have been developed to maintain such metrology, as described by Butler et al (1996) and Lovis et al (2006). Figure 1 shows the velocities of a representative 'standard' star, HD 9407 observed with the HIRES spectrometer at the Keck telescope (Vogt et al 1994). The velocity scatter of $1.37 \mathrm{~m} \mathrm{~s}^{-1}$ during the past 8 years represents a total error budget empirically of less than $1.5 \mathrm{~m} \mathrm{~s}^{-1}$ (including all noise sources and any signals from small planets). Velocity fields on the surfaces of Solar-type stars will limit secure planet-detection at the $1 \mathrm{~m} \mathrm{~s}^{-1}$ level, corresponding to planets of 10 Earth-masses at $1 \mathrm{AU}$.

The various Doppler groups have now surveyed $\sim 2500$ stars having masses $0.3-2.5 M_{\odot}$ and being brighter than

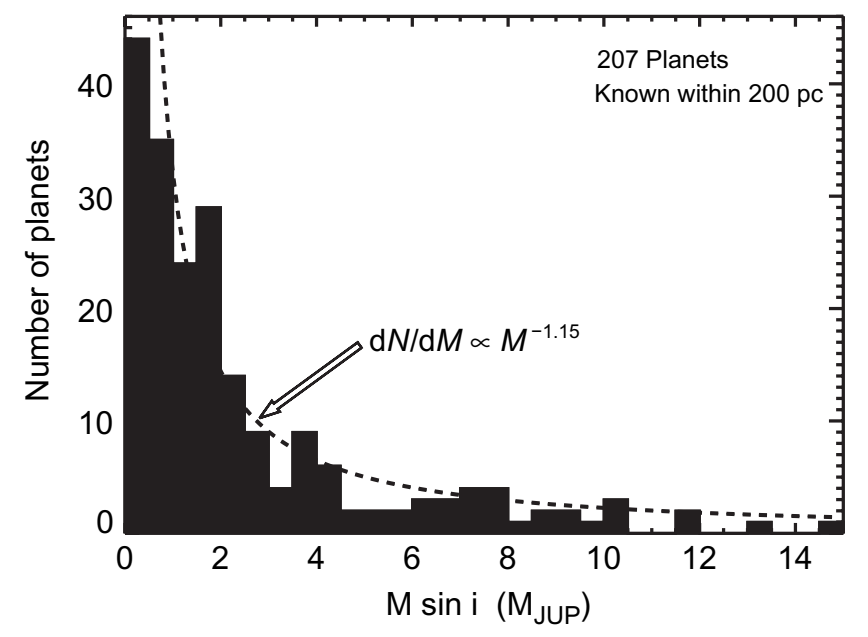

Figure 2. The mass distribution of planets around main-sequence stars from 0.3 to 2.0 solar masses. Planet masses range from 5 Earth masses (Gliese $876 \mathrm{~d}$ and Gliese $581 \mathrm{c}$ ) to as high as 15

Jupiter-masses.

magnitude, $V=12$. Binary stars separated by less than 2 arcsec and magnetically active stars younger than 1 Gyr are usually avoided in the surveys. Our CCPS group has published a list of target stars (Wright et al 2004) to permit statistical analyses by others, e.g. Cumming et al (2008).

About $87 \%$ of our stars surveyed for at least a decade show no Doppler variation at all at a $3-\sigma$ limit of $10 \mathrm{~m} \mathrm{~s}^{-1}$. For them, Jupiter-mass planets within $\sim 3 \mathrm{AU}$ are unlikely, as are Saturn-mass planets within $1 \mathrm{AU}$. About $6 \%$ of the stars reveal long term trends in radial velocity, often with curvature, indicative of a companion having a planetary, brown dwarf, or (low mass) stellar nature with an orbital period of a decade or more (Patel et al 2007). These stars constitute a rich mine of low mass companions available for direct imaging and spectroscopy with coronagraphic cameras, with ages and metallicities known in advance from the host star. About 7\% of the stars reveal clear Keplerian signatures of planets, with 250 planet candidates found by all teams.

\section{Masses and orbits of exoplanets}

Of the known planet candidates, 207 have secure orbital information permitting statistical assessment. They have minimum masses $(M \sin i)$ between 0.1 and $\sim 15 M_{\mathrm{JUP}}$ and orbit between 0.02 and $6 \mathrm{AU}$. The distribution of minimum masses is shown in figure 2. The mass distribution of exoplanets rises toward smaller masses down to Saturn masses without question. A power law fit gives $\mathrm{d} N / \mathrm{d} M \propto M^{-1.15}$. The incompleteness is greatest at small masses indicating that the mass distribution may rise even more steeply toward lower masses.

The distribution of semimajor axes, $a$, rises (in logarithmic bins) as $\mathrm{d} N / \mathrm{d} \log a \propto a^{+0.4}$, as shown in figure 3 . There is still a pile-up of planets at $0.05 \mathrm{AU}$ and an apparent minimum at $0.3 \mathrm{AU}$. The decline beyond $3 \mathrm{AU}$ is likely due to the limited duration, $\sim 10$ years, of Doppler surveys. Integration out to $20 \mathrm{AU}$ (similar to Uranus' orbit) requires extrapolation as few stars have been surveyed long enough to detect planets beyond $5 \mathrm{AU}$. Such extrapolation 


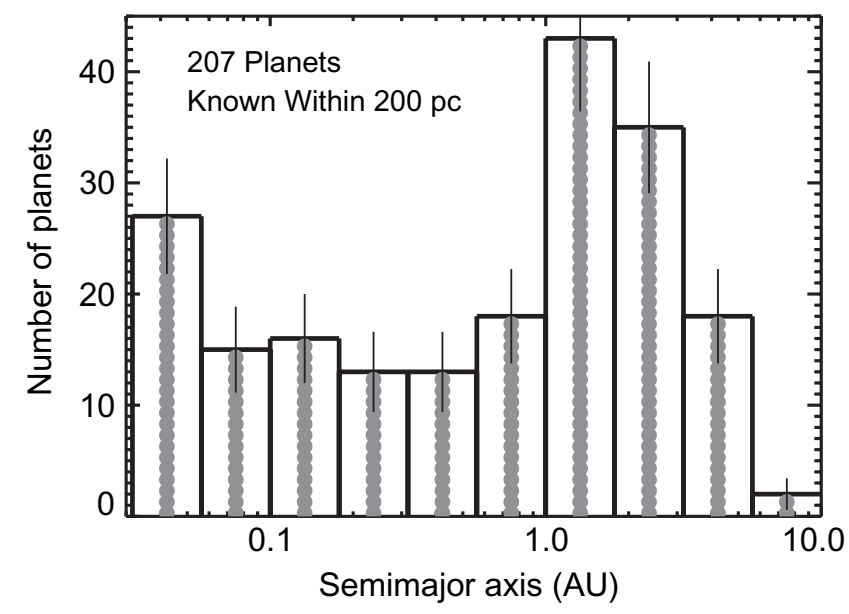

Figure 3. Distribution of semimajor axes of exoplanets. There is a clear rise toward larger orbital distances. Extrapoloating, the total occurrence is $19 \%$ for Saturn and Jupiter-mass planets within $20 \mathrm{AU}$.

and integration suggests that $19 \%$ of Sun-like stars have a giant planet within $20 \mathrm{AU}$ (Cumming et al 2008). The observed masses and orbits of giant planets within $5 \mathrm{AU}$ are reproduced fairly well by current theories of their formation and migration (Kley et al 2005) including their dependence on stellar metallicity and mass as shown by Ida and Lin (2004), Ida and Lin (2005) and Armitage (2007).

Beyond $20 \mathrm{AU}$, gas giants may be rare according to current theory (that has a dubious history of success). The decline with radius of the gas densities of protoplanetary disks along with longer dynamical timescales there imply a longer timescale for planet growth beyond $20 \mathrm{AU}$, which still must compete against a short protoplanetary disk lifetime, $\sim 3 \mathrm{Myr}$, e.g. Alibert et al (2005) and Hubickyj et al (2004). Efforts to image giant planets around young stars from their thermal infrared (IR) emission have yielded upper limits on their occurrence, suggesting a declining occurrence beyond 30 AU, e.g. Apai et al (2007) and Lafreniere et al (2007).

The orbital eccentricities of exoplanets range from circular to $e=0.93$ with a broad distribution, as shown in figure 4 . The planets orbiting within $0.05 \mathrm{AU}$ reside mostly in nearly circular orbits, as expected due to tidal circularization. There is a hint of a subtle rise in the upper envelope of eccentricities between 0.05 and 1.0 AU that may be related to tidal circularization or orbit decay at periastron for orbits of highest eccentricity, either currently or during the pre-main-sequence lifetime of the star. Most explanations for the origin of the orbital eccentricity involve the mutual gravitational interactions of planets (or planetesimals) with each other, either via gentle, incremental perturbations, resonances, or deep scattering, e.g. Marzari et al (2005), Juric and Tremaine (2007), Chatterjee et al (2007), Ford and Rasio (2007), Fabrycky and Tremaine (2007), Ford and Chiang (2007).

\section{Exoplanet interiors: heavy element enhancement and cores}

The majority of the 250 exoplanets discovered thus far are likely gaseous, made primarily of $\mathrm{H}$ and $\mathrm{He}$, as demonstrated

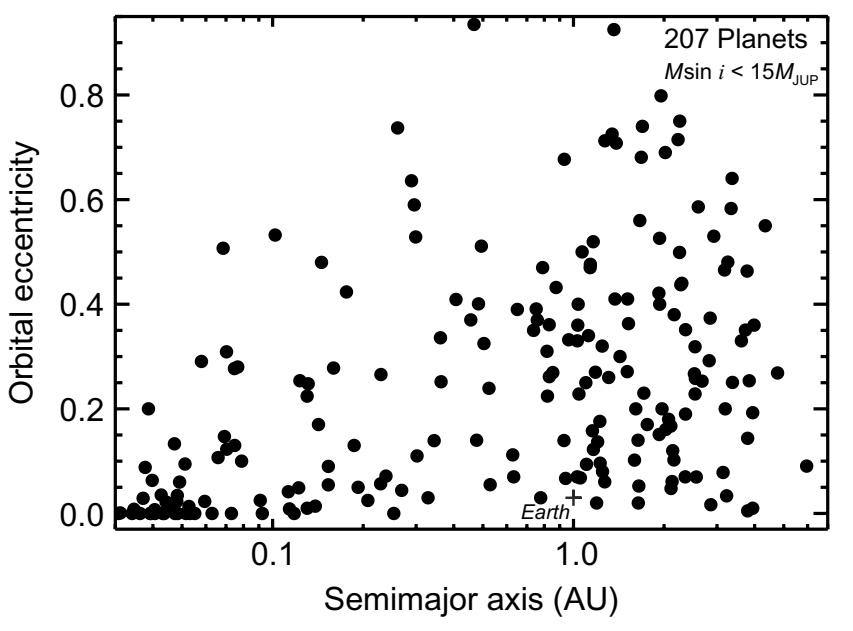

Figure 4. Orbital eccentricities versus semimajor axis (on a log scale) of the 207 known exoplanets. The close-in planets are tidally circularized. Remarkably, the planets between 2.5 and 4.0 AU that approach the orbital distance of Juptier (5.2 AU) still exhibit a large range of orbital eccentricities, suggesting that the circular orbits are not the rule for giant planets such as Jupiter. Thus, circular orbits may not be the rule for planetary systems, rendering the Solar System somewhat unusual.

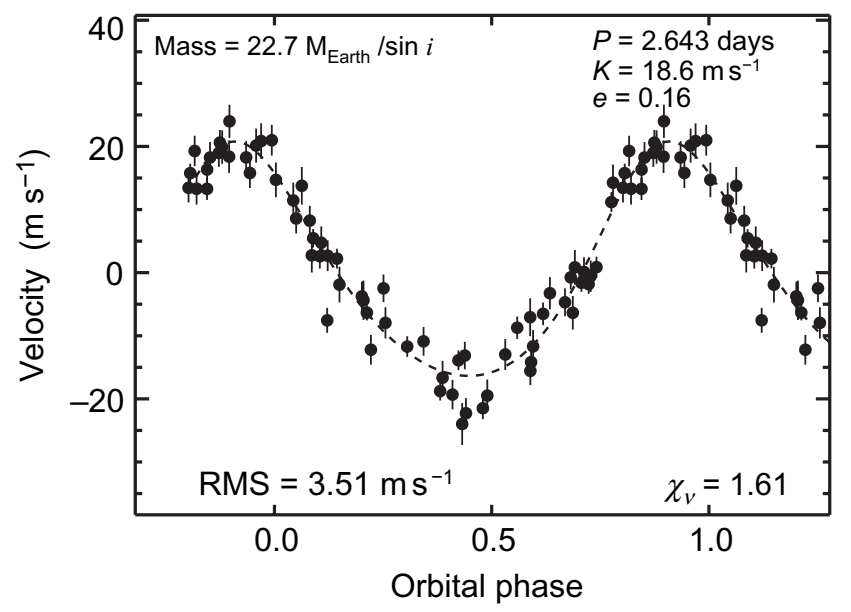

Figure 5. Velocity versus orbital phase for Gliese 436 exhibiting the planet of 22.7 $M_{\text {Earth. }}$. The transit detected by Gillon et al (2007) and remeasured by Deming et al (2007) shows its radius is 3.95-4.4 Earth radii, implying a density of $2.0-1.5 \mathrm{~g} \mathrm{cc}^{-1}$.

convincingly from their measured densities of $\sim 1 \mathrm{gm} \mathrm{cm}^{-3}$ revealed by the transiting planets. For two planets, HD 149026 and Gliese 436, the measured radii are so small that the $\mathrm{H}$ and He must be greatly enriched by large amounts of heavy elements (Gillon et al 2007, Sato et al 2005).

The mass of the planet orbiting Gliese 436 is $22.7 \pm$ 1.0 $M_{\text {Earth }}$ from the HIRES velocity measurements shown in figure 5 (Butler et al 2004, Maness et al 2007, Vogt et al 1994). The measured radius of $4.33 R_{\text {Earth }}$ implies a density of $1.55 \mathrm{~g} \mathrm{~cm}^{-3}$ that is most easily explained by an interior consisting of an iron-nickel and silicate core surrounded by an envelope of ice composed of water, methane, ammonia and a thick shell of $\mathrm{H}-\mathrm{He}$, as shown in figure 6 (Deming et al 2007). There can be little doubt that this planet's interior has a mean molecular weight well above that provided by $\mathrm{H}$ and $\mathrm{He}$, implying a large contribution of heavy elements, similar to the transiting planet HD 149026 (Gillon et al 2007, Sato et al 2005). Knowledge of mass and radius leaves 


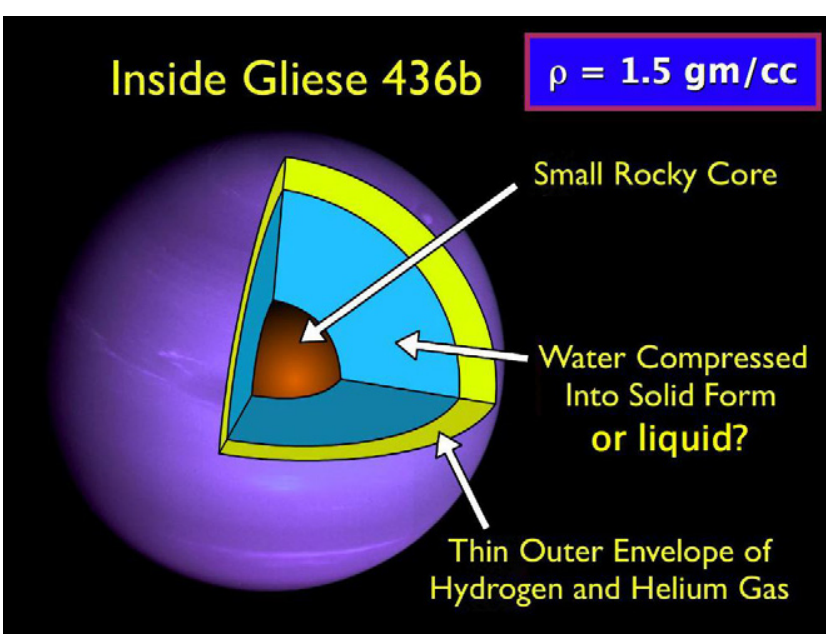

Figure 6. Internal structure of Gliese 436 . The measured density of $1.55 \mathrm{~g} \mathrm{~cm}^{-3}$ can be explained by a iron and rocky core surrounded by an envelope of water and methane ice and an outer layer of $\mathrm{H}-\mathrm{He}$, implying it formed beyond the snow line at $\sim 1 \mathrm{AU}$ (thanks to Eugene Chiang for this calculation). Planets of 5-25 $M_{\text {Earth }}$ may have a similar composition if they form beyond the snow line (see contribution by Sasselov et al 2008).

degeneracy in models between the relative contributions of iron-nickel, silicates, ices and H-He (David Stevenson 2007, private communication).

\section{Comparisons of planetary systems to our Solar System}

A rational anthropocentric question centers on whether our Solar System is a common or unusual type of planetary system in structure and chemical composition, with implications for an anthropic principle in planetary science. Our Solar System could have unusual attributes if our existence were contingent upon them. Prior to 1995, most theoretical models predicted planetary systems to be similar to our Solar System, including co-planar, circular orbits and gas giants orbiting beyond $5 \mathrm{AU}$ and rocky planets closer in. Our discovery that most exoplanetary systems have giant planets in more eccentric orbits, at least within $5 \mathrm{AU}$, suggest that common perturbative processes may cause non-circular orbits for planets in general, including those beyond $5 \mathrm{AU}$ and rocky planets closer in. Giant planets in non-circular orbits tend to eject smaller planets, such as the Earth-sized ones, and they induce eccentricities in planets forcing them to suffer variations in stellar flux that puts the annual stability of liquid water in jeopardy.

The one planet in our Solar System amenable to direct comparison with exoplanets is Jupiter at 5.2 AU. Two known exoplanets are its closest analog. The star 55 Cancri (with mass $0.94 M_{\odot}$ ) has a planet, ' $55 \mathrm{Cnc}$ d', with minimum mass 3.8 $M_{\text {JUP }}$ orbiting with semimajor axis 5.8 AU. This is the only exoplanet having a securely known orbit that resides farther from its star than Jupiter. The eccentricity of $55 \mathrm{Cnc} \mathrm{d}$ is $e=$ $0.06 \pm 0.03$, nearly circular, and it has four planets orbiting inward of it, all lower mass, reminiscent superficially of our Solar System. The 5th planet at $0.8 \mathrm{AU}$ was only recently established, as shown in figure 7 from Fischer et al (2008). This is the only planetary system known with five planets.

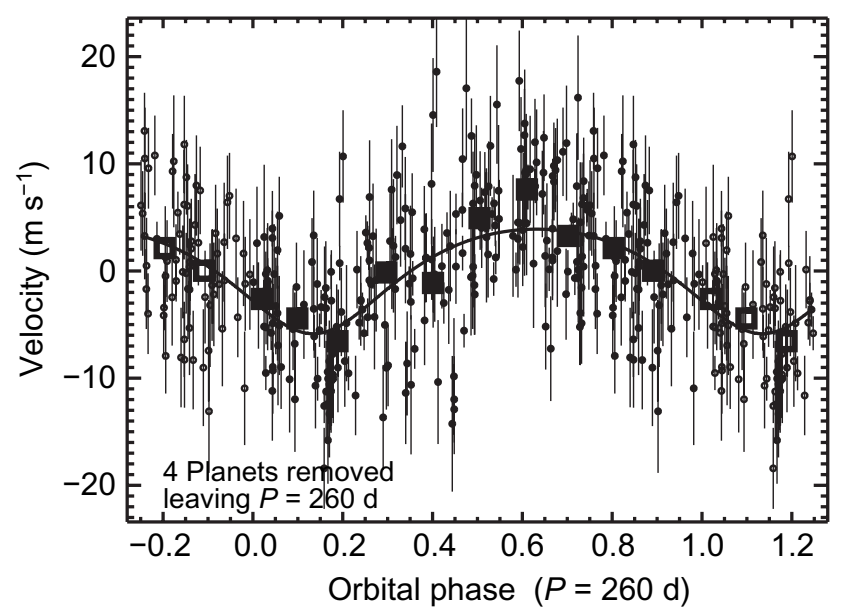

Figure 7. Residual velocities versus orbital phase for 55 Cancri (for $P=260$ d) after the velocities induced by the four other planets are subtracted. The orbital parameters were established with a simultaneous 5-planet Keplerian fit to all Doppler measurements. The residual velocities reveal the periodic variation associated with the new planet. The solid line shows the Keplerian curve of the $260 \mathrm{~d}$ planet alone. The planet's minimum mass is $45 M_{\text {Earth }}$ and the semimajor axis is $0.78 \mathrm{AU}$ (Fischer et al 2008).

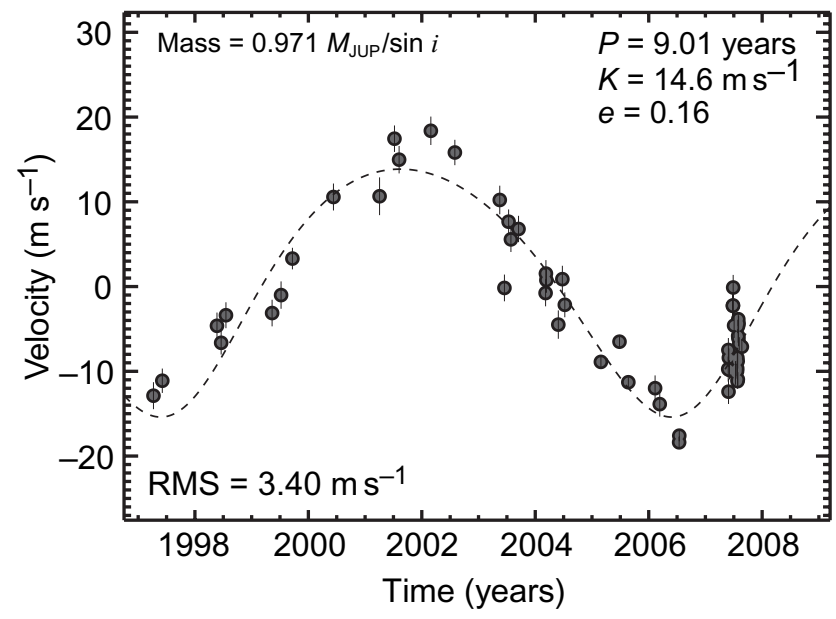

Figure 8. Doppler measurements versus time for the G8 V star, HD 154345 , showing a planet having a minimum mass of $0.9 M_{\mathrm{JUP}}$ orbiting 4.14 AU from the star. The orbit is nearly circular and there are no discernable giant planets inward of it, making this the closest extrasolar analog to Jupiter (Wright et al 2007).

But the 55 Cnc system is different from the Solar System in two respects. The most massive planet is likely $4-6 M_{\mathrm{JUP}}$ (accounting for $\sin i$ ) and there are four large planets, not small rocky planets, orbiting inward of $1 \mathrm{AU}$.

The best analog of Jupiter is a recently discovered exoplanet orbiting HD 154345 (Wright et al 2007), a star with a mass of $0.88 M_{\odot}$ and spectral type, G8 V. Its Doppler signal from Keck-HIRES is shown in figure 8, revealing a planet with a minimum mass of $0.92 M_{\mathrm{JUP}}$ in an orbit with a period $P=9.0$ year, semimajor axis $a=4.2$ AU and eccentricity $e=$ $0.16 \pm 0.06$. This planet has a mass likely similar to that of Jupiter residing in a nearly circular orbit, and it has no giant planets inward of it. There is growing evidence of additional planets at 45 or $60 \mathrm{~d}$, requiring more velocity measurements. The star itself has an abundance of heavy elements within $30 \%$ that of the Sun, $[\mathrm{Fe} / \mathrm{H}]=-0.10 \pm 0.04$ from Valenti and Fischer (2005). 


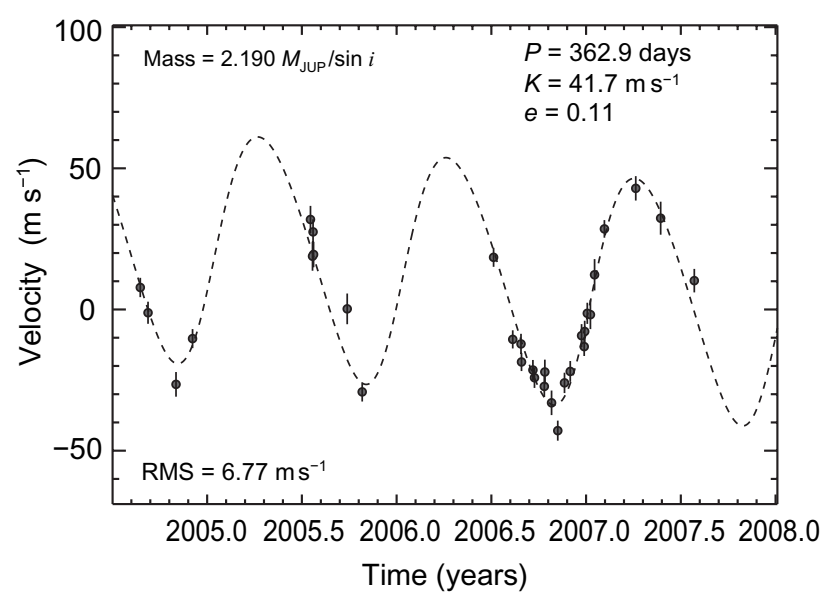

Figure 9. Velocity versus time for a representative evolved star HD 210702 having a well defined stellar mass of $1.85 M_{\odot}$. The velocities are well fit by a Keplerian model and linear trend (dashed line) with $P=362.9 \mathrm{~d}, K=41.7 \mathrm{~m} \mathrm{~s}^{-1}$ and $M \sin i=2.2 M_{\mathrm{JUP}}$ (Johnson et al 2007).

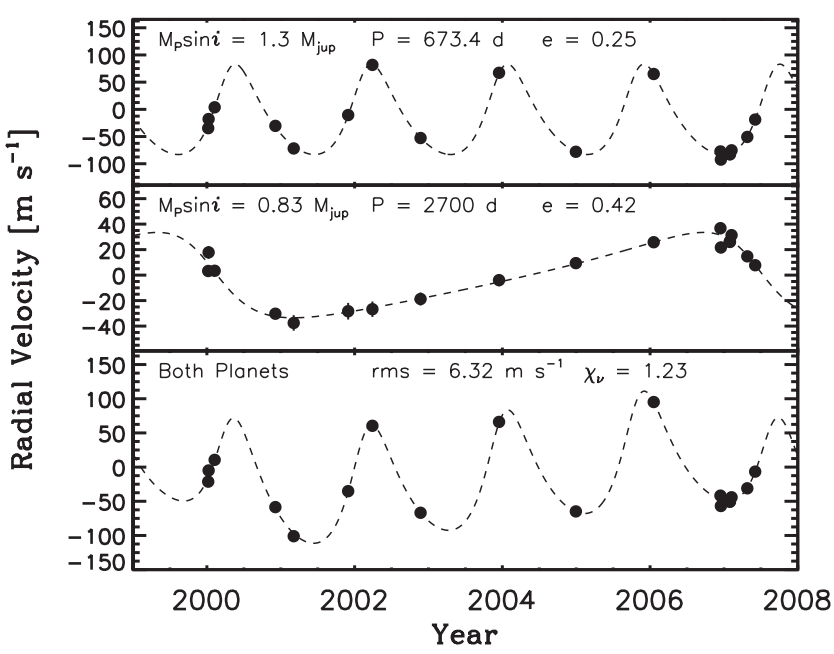

Figure 10. Doppler measurements versus time for the $M$ dwarf, Gliese 317, showing two planets of Jupiter mass. The bottom panel shows the measured velocities (dots) and the two-planet model (dashes). The upper two panels show the measured velocities and model with the other planet removed. Jupiter-mass planets occur less often around low-mass stars than solar-type stars (Johnson et al 2007).

\section{Exoplanets as a function of stellar mass}

We have extended the Doppler planet search to stars having masses up to $2 M_{\odot}$ and as low as $0.3 M_{\odot}$ to sense the dependence of planet properties on stellar mass. Doppler measurements of $G$ and K-type subgiants that had been early $\mathrm{F}$ and A-type main-sequence stars permit detection of planets around stars of 1.5-2.5 $M_{\odot}$ (Johnson et al 2007). As an example, HD 210702 is a subgiant of mass of $1.85 M_{\odot}$ for which the Doppler measurements during the past 3 years are shown in figure 9. Similarly, a half dozen giant planets have now been detected around stars with masses between 1.5 and $2.5 M_{\odot}$ (Johnson et al 2007), yielding a detection rate of $8.5 \%$ for Jovian planets within 2.5 AU, apparently higher than that for Solar-type stars $(4 \%)$.

In contrast, $\mathrm{M}$ dwarfs harbor few Jupiter-mass planets. Figure 10 shows a rare case of an M dwarf, Gliese 317 ,

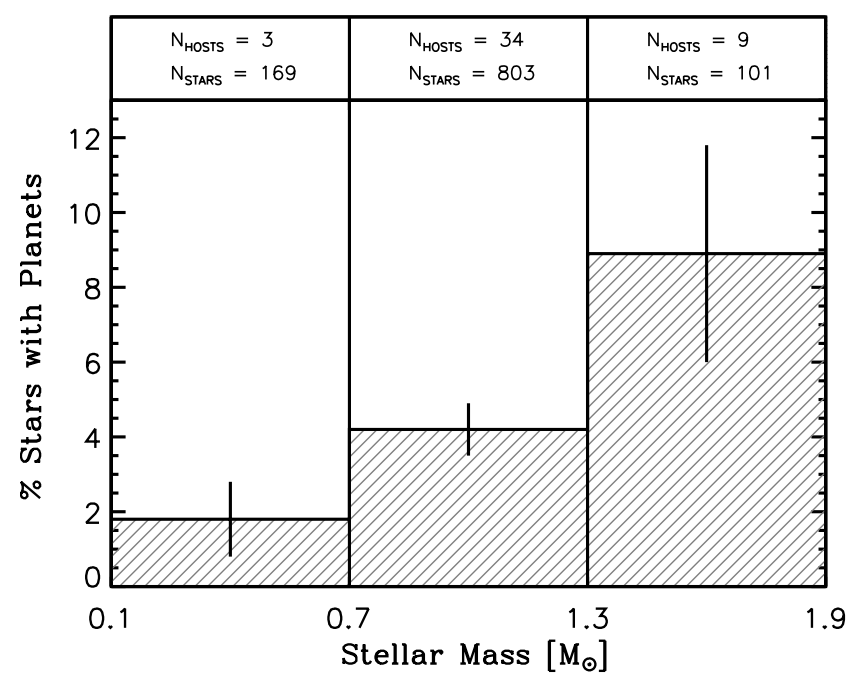

Figure 11. Planet occurrence versus stellar mass for giant planets within $2.5 \mathrm{AU}$. Low mass stars have fewer jupiters, while high mass stars above 1.3 solar masses have more. Future work must account for metallicity biases (Johnson et al 2007).

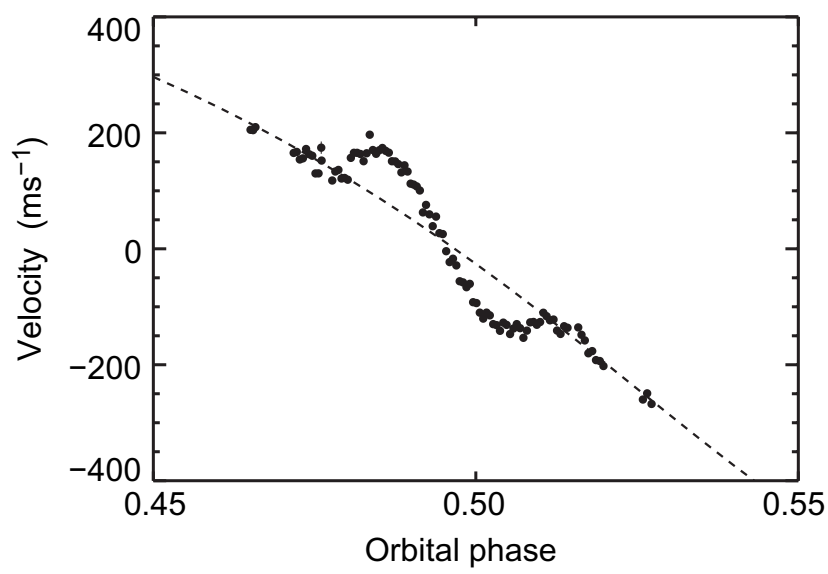

Figure 12. Velocity versus orbital phase for HD 147506 (HAT-P-2b) during the transit of the planet. The dashed line is the stellar velocity predicted from the wobble of the star. The wave-shaped departure of the velocities is due to the Rossiter-McLaughlin effect as the planet alternately blocks the approaching and then receding sides of the rotating star's hemisphere. The symmetry of the departure shows that the planet crosses the star in a path parallel to (within $13^{\circ}$ of) the stellar equator in the direction of stellar spin, unexpected from the high eccentricity, $e=0.5$, of the planet (Winn et al 2007).

that has two Jovian planets with minimum masses of 0.83 and $1.3 M_{\mathrm{JUP}}$. The occurrence of jupiters around $\mathrm{M}$ dwarfs is only $2 \%$. Figure 11 shows the occurrence frequency of Jupiter-mass planets around stars in three stellar mass bins, $0.1-0.7,0.7-1.3$ and $1.3-1.9 M_{\odot}$. The occurrence of gas giants increases with stellar mass. As there could be selection effects involving metallicity, this result must be confirmed with more detections including a range of stellar metallicities.

\section{The Rossiter-McLaughlin effect and multi-planet systems}

A remarkable transiting planet, HD 147506 b, was discovered by photometric dimming (Bakos et al 2007). The eccentricity 


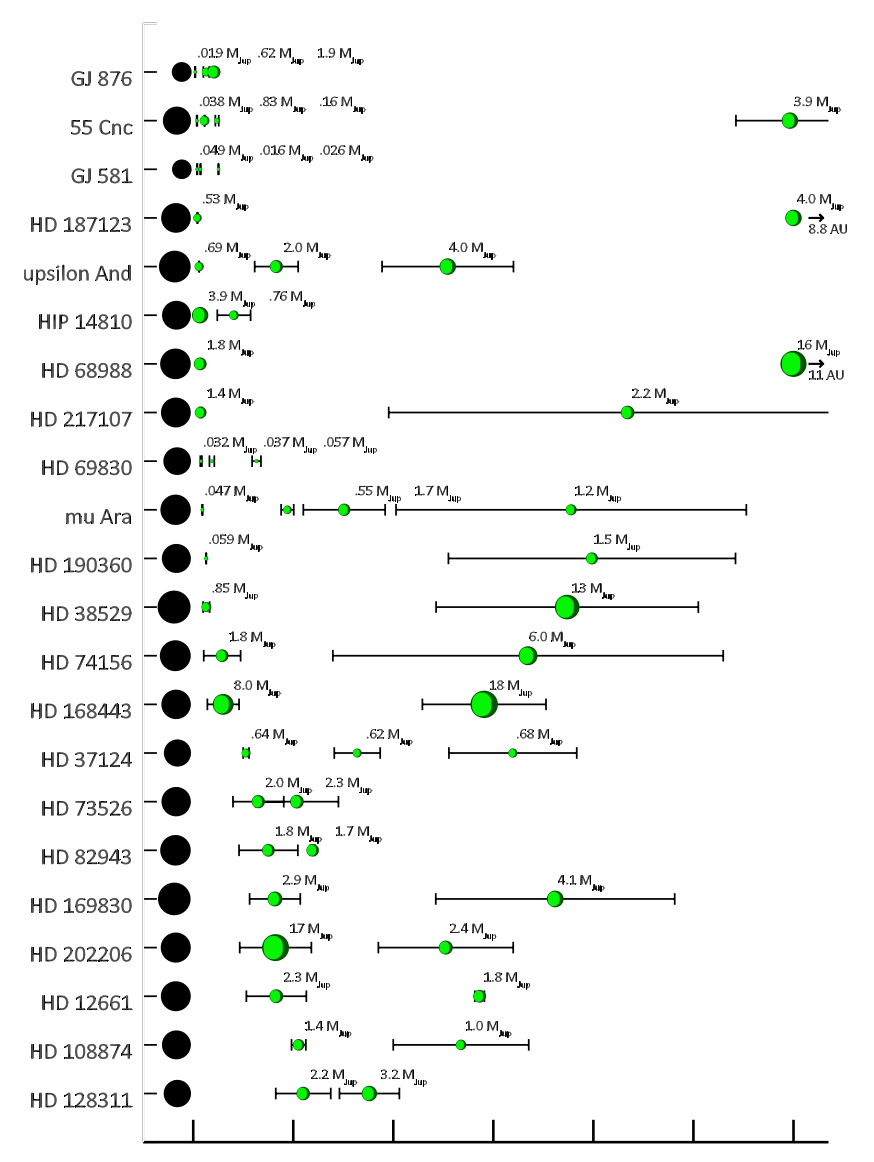

$\begin{array}{lllllll}0 & 1 & 2 & 3 & 4 & 5 & 6\end{array}$

\section{Semimajor Axis (AU)}

Figure 13. The 22 multi-planet systems with well-defined orbital parameters. For each system the star is at left shown with a filled circle having diameter proportional to the mass of the star. At the right are the orbiting planets, with masses noted. The horizontal bar through each point represents the closest and farthest approach of the planet from the star. Four systems are in mean motion resonances.

of $e=0.5$ is the highest of any known close-in exoplanet and defies the expected tidal circularization. The high eccentricity suggests that the planet suffered a significant scattering event on a timescale less than the tidal circularization time. Such a scattering could result in an orbital plane disturbed from the coplanarity expected for nascent planetary systems including the stellar equator.

The transiting planet alternately blocks the approaching and then receding sides of the rotating star's hemisphere, the 'Rossiter-McLaughlin effect,' offering an opportunity to sense the geometry of the orbit relative to the stellar spin (Winn 2007a). Figure 12 shows the apparent symmetry of the departure stellar velocities for HD 147506, indicating that the planet crosses the star in a path parallel to the stellar equator, and that the orbital angular momentum and star's spin angular momentum are aligned (Winn et al 2007). Such coplanarity is not expected if gravitational scattering or Kozai resonance brought the planet close to the star (Chatterjee et al 2007, Fabrycky and Tremaine 2007, Winn 2007b).

The puzzling orbit of HD 147506 suggests that planet-planet interactions shape the architectures of planetary systems. To date, 22 multi-planet systems have well-defined minimum masses and orbits, as shown in figure 13. Four of the systems exhibit mean motion resonances. As their original orbits had arbitrary orbital periods, the planets must have migrated into their current traps. Notably, most hot jupiters, with periods under 10 days, do not have another giant planet within 3 AU. Further work is needed to understand if Kozai interactions among planets followed by tidal circularization can explain the hot jupiters.

\section{Acknowledgments}

We thank Jack Lissauer, Eric Ford, Eugene Chiang, Jonathan Fortney, Greg Laughlin and Frank Selsis for valuable conversations. We thank the Lick, Keck and Anglo-Australian Observatories for their outstanding technical and administrative work to design, build and maintain the spectrometers, data-taking systems and telescopes, without which measuring Doppler shifts with a precision of $1 \mathrm{~m} \mathrm{~s}^{-1}$ would be impossible. We thank the NASA, the National Science Foundation, the UK Science and Technology Facilities Council, the Australian Research Council, and STFC and DETYA for funding that makes this research possible.

\section{References}

Alibert Y, Mordasini C, Benz W and Winisdoerffer C 2005 Astron. Astrophys. 434 343-53

Apai D, Meyer M R, Hinz P and Kasper M 2007 Preprint 710

Armitage P J 2007 Astrophys. J. 665 1381-90

Bakos G A et al 2007 Astron. Astrophys. 670 826-32

Butler R P, Marcy G W, Williams E, McCarthy C, Dosanjh P and Vogt S S 1996 Publ. Astron. Soc. Pacific 108500

Butler R P, Vogt S S, Marcy G W, Fischer D A, Wright J T, Henry G W, Laughlin G and Lissauer J J 2004 Astrophys. J. 617 $580-8$

Chatterjee S, Ford E B and Rasio F A 2007 Astrophysics Preprint

Cumming A, Butler R P, Marcy G W, Vogt S S, Wright J T and Fischer D A 2008 PASP in press

Deming D, Harrington J, Laughlin G, Seager S, Navarro S B, Bowman W C and Horning K 2007 Astrophys. J. 667 L199-202

Fabrycky D and Tremaine S 2007 Astrophys. J. 664 1298-1315

Fischer D A et al 2008 Astrophys. J. 675 790-801

Ford E B and Chiang E I 2007 Astrophys. J. 661 602-15

Ford E B and Rasio F A 2007 Astrophysics Preprint

Gillon M, Pont F, Demory B O, Mallmann F, Mayor M, Mazeh T, Queloz D, Shporer A, Udry S and Vuissoz C 2007 Astron. Astrophys. 472 L13-6

Hubickyj O, Bodenheimer P and Lissauer J J 2004 Revista Mexicana de Astronomia y Astrofisica Conf. Ser. pp 83-6

Ida S and Lin D N C 2004 Astrophys. J. 616 567-72

Ida S and Lin D N C 2005 Astrophys. J. 626 1045-60

Johnson J A, Butler R P, Marcy G W, Fischer D A, Vogt S S, Wright J T and Peek K M G 2007 Astrophys. J. 670 833-40

Johnson J A, Fischer D A, Marcy G W, Wright J T, Driscoll P, Butler R P, Hekker S, Reffert S and Vogt S S 2007 Astrophys. J. $665785-93$

Juric M and Tremaine S 2007 Astrophysics Preprint

Kley W, Lee M H, Murray N and Peale S 2005 Astron. Astrophys. $437727-42$

Lafreniere D et al 2007 Astrophys. J. 670 1367-90 
Lovis C, Pepe F, Bouchy F, Lo Curto G, Mayor M, Pasquini L, Queloz D, Rupprecht G, Udry S and Zucker S 2006 Ground-based and Airborne Instrumentation for Astronomy (Proc. SPIE vol 6269) ed I S McLean and M Iye, p 62690P

Maness H L, Marcy G W, Ford E B, Hauschildt P H, Shreve A T, Basri G B, Butler R P and Vogt S S 2007 Publ. Astron. Soc. Pacific 119 90-101

Marzari F, Weidenschilling S J, Barbieri M and Granata V 2005 Astrophys. J. 618 502-11

Patel S G, Vogt S S, Marcy G W, Johnson J A, Fischer D A, Wright $\mathrm{J}$ T and Butler R P 2007 Astrophys. J. 665 744-53

Sasselov D D, Valencia D and O'Connell R J 2008 Phys. Scr. T130 014035
Sato B et al 2005 Astrophys. J. 633 465-73

Valenti J A and Fischer D A 2005 Astrophys. J. Suppl. 159 141-66

Vogt S S et al 1994 Proc. SPIE Instrum. in Astronomy VIII vol 2198, ed D L Crawford and E R Craine, p 362

Winn J N 2007a Transiting Extrapolr Planets Workshop 366

(Astronomical Society of the Pacific Conference Series) ed

C Afonso, D Weldrake and T Henning pp 170

Winn J N 2007 b Preprint 710

Winn J N et al 2007 Astrophys. J. 665 L167-70

Wright J T, Marcy G W, Butler R P and Vogt S S 2004 Astrophys. J. Suppl. 152 261-95

Wright J T et al 2007 Astrophys. J. 657 533-45 